\section{EXPERIENCIA INNOVADORA \\ EN LA DOCENCIA DE \\ LAS ASIGNATURAS DEL \\ GRADO DE INGENIERÍA \\ DE DISEÑO INDUSTRIAL Y \\ DESARROLLO DE PRODUCTO \\ BASADA EN ACTIVIDADES \\ MULTIDISCIPLINARES}

\author{
Ramón Miralbes, José Manuel Auria \\ Enrique Tardío, Ignacio López \\ Departamento de Ingeniería de Diseño y Fabricación, \\ Universidad de Zaragoza \\ Ed. Torres Quevedo, C/ María de Luna s/n (500017 Zaragoza) \\ miralbes@unizar.es
}

\begin{abstract}
The aim of this article is the presentation of the obtained results and the developed methodology used in an innovative teaching project where there was defined, developed and implemented a multidisciplinary activity that bring together diverse matters of a semester and the developed knowledge's of all the matters in only one project. With this methodology the objective is that all the matters and their professors work together to obtain the same global objectives.
\end{abstract}

KEY WORDS: Multidisciplinary; innovative; teaching; project; module.

\section{INTRODUCCIÓN}

Esta ponencia se presenta como resultado de un proyecto de innovación docente desarrollado para las asignaturas del primer cuatrimestre del segundo curso del grado de Ingeniería de Diseño Industrial y Desarrollo del Producto. Las asignaturas integrantes en el proyecto son Mecánica, Expresión Gráfica II, Diseño Asistido por Ordenador y Taller Il y se desarrolla según las indicaciones mostradas en el desarrollo del curso de "diseño y evaluación de proyectos de innovación docente" impartido en el ICE de la UZ por Tomás Escudero Escorza en febrero de 2010. Se evalúa

\section{INNOVATIVE TEACHING EXPERIENCE IN THE INDUSTRIAL DESIGN AND PRODUCT DEVELOPMENT ENGINEERING DEGREE USING MULTIDISCIPLINARY ACTIVITIES}

RESUMEN: El objetivo de este artículo es la presentación de los resultados obtenidos y de la metodología desarrollada durante un proyecto de innovación docente cuyo objetivo era la definición, desarrollo e implantación de un proyecto de multidisciplinar que aglutine las diversas asignaturas de un cuatrimestre y en el que se desarrollen los conocimientos adquiridos en cada una de ellas. Se pretende con esta metodología que todas las asignaturas participen en la consecución de unos objetivos globales.

PALABRAS CLAVE: Multidisciplinar; innovación; docencia; proyecto; módulo.

según el criterio de por qué se desarrolla el proyecto, que acciones se llevan a cabo y de qué manera y por último la evaluación de los resultados del proyecto, los actuales y a futuro los que podamos valorar.

La innovación radica en la definición de un proyecto común para desarrollar de forma conjunta en las asignaturas involucradas, aglutinando los conocimientos adquiridos en cada una de ellas. Se pretende con esta metodología de trabajo por módulos que todas las asignaturas participen conjuntamente en la consecución de unos objetivos globales. El proyecto se realiza en grupos de cuatro alumnos. 


\section{Objetivos}

Como objetivo general del módulo se pretende conseguir que los alumnos al finalizar el mismo sean capaces de hacer análisis, obtener conclusiones y plantear mejoras de producto de manera que estas puedan tener una representación final. Este objetivo se ha conseguido, se han desarrollado esas actividades dentro del trabajo común de modo satisfactorio, salvo en algunos casos aislados en los que los alumnos han mostrado disconformidad en algún aspecto del proceso de trabajo, pero en general todos los alumnos que han desarrollado y terminado el trabajo de módulo han conocido y son capaces de desarrollar el proceso de diseño, completar sus fases y actividades, desde la documentación hasta la presentación de producto.

Se pretende con esta forma de trabajo por módulos que todas las asignaturas participen en la consecución de unos objetivos globales de la siguiente forma, las asignaturas se agrupan según los objetivos perseguidos: Taller de Diseño II y Mecánica participaran de la parte de análisis, obtención de conclusiones y aportación de mejoras de producto y Expresión Gráfica II y Diseño Asistido por Ordenador (DA0) participaran en la representación de esta mejora.

Cada asignatura cuenta con unos objetivos propios que son los que permiten conseguir los objetivos del módulo y que son los evaluados para la superación de la asignatura.

Otro de los objetivos perseguidos con esta innovación es reducir la carga de trabajo del estudiante de forma que desarrollando un único tema, tareas como la búsqueda de información, el estudio y cálculo mecánico, la presentación bi y tridimensional del producto, que anteriormente se realizaban de forma independientemente para cada una de las asignaturas, se van a realizar de forma conjunta.

Otra de las metas planteadas era la evaluación continua y conjunta, en la que el proyecto se valora en diferentes fases de su elaboración por el grupo de profesores. Para ello se ha realizado una planificación en la secuenciación de entregas parciales en las distintas asignaturas, teniendo en cuenta que el flujo de actividades no es uniforme en cada una de las asignaturas sino que se adapta a las exigencias marcadas por el desarrollo natural del proyecto. También se realiza una evaluación conjunta por parte del grupo de profesores del módulo, dando de este modo una nota global al trabajo del grupo de alumnos que tendrá mismo el peso en el global de cada una de las asignaturas.

\section{Desarrollo}

Las principales actividades llevadas a cabo en el proyecto han sido: planificación y programación del proyecto de innovación docente, implantación de la nueva metodología de trabajo, gestión del proyecto durante el desarrollo, evaluación de los resultados de los trabajos, evaluación de los resultados generales del proyecto e identificación de puntos débiles y propuestas de mejora.

En general se puede decir que las actividades desarrolladas en el proyecto se han cumplido satisfactoriamente y que este proyecto está en una fase inicial de puesta en marcha, siendo necesario hacer varias cambios y correcciones en las actividades desarrolladas en sucesivos cursos para poder evaluar correctamente y plantear las acciones de mejora pertinentes.

\section{Evaluación del trabajo de módulo}

La evaluación del trabajo de módulo se ha realizado mediante una presentación oral ante el tribunal de profesores, compuesto por al menos un profesor de cada asignatura con una extensión máxima de veinte minutos de exposición más diez minutos de preguntas y mediante una revisión de los informes presentados por los estudiantes.

Se ha planteado que la nota del módulo sea común para todos los integrantes del grupo y que, para cada una de las asignaturas involucradas, repercuta de la misma forma en la evaluación global. Así pues, para cada asignatura se ha planteado que un $30 \%$ de la nota global sea la nota del trabajo de módulo. De este 30\%, un 10\% corresponde a la nota común de módulo, establecida por el tribunal de módulo tras la presentación y la evaluación conjunta del trabajo de módulo y el otro $20 \%$ corresponda a la evaluación por parte de cada uno de los profesores de la parte correspondiente del trabajo de módulo relacionado con la asignatura correspondiente. 
Se ha planteado que sea necesario para aprobar cada una de las asignaturas, como requisito indispensable, una nota mínima en el trabajo de módulo de 5.00. Con ello se busca una coherencia entre los criterios de valoración y de calificación entre las diversas asignaturas.

Durante el primer año de implantación del módulo, se solicitó un único informe común en papel de todas las partes involucradas; este aspecto implicó por un lado un elevado desembolso económico para los alumnos (debido a la amplia extensión del trabajo) y por otro lado una gran dificultad por parte de los profesores para realizar la valoración del trabajo de módulo referente a cada asignatura, ya que solo se disponía de una única copia del trabajo. Para solucionarlo se implantó la herramienta para la entrega telemática del trabajo y se dividió el trabajo en cuatro entregas, una para cada asignatura del módulo, con lo que se reduce el gasto y además se presentan informes diferentes para cada asignatura, pero cualquier profesor puede ver los informes del resto de asignaturas y valorar aspectos multidisciplinares y transversales.

\section{ANÁlisIS de RESULtados}

\section{Informe del grupo de profesores sobre el trabajo en modulo}

En la elaboración de este proyecto de innovación docente se han detectado una serie de puntos fuertes y débiles, de los que se pueden definir acciones de mejora con sus condicionantes. Además de una serie de conclusiones tanto del proyecto en conjunto como de cada una de las asignaturas.

\section{Puntos fuertes}

Al inicio del cuatrimestre de hace una presentación conjunta del módulo y de todas las asignaturas, en esta presentación se exponen las ventajas del trabajo en módulo y de cómo cada asignatura aporta una parte importante al trabajo común de módulo.

En la presentación de módulo se hacen los grupos de prácticas y subgrupos para hacer los diferentes trabajos de asignatura y módulo. Con los grupos hechos de esta manera no hay descoordinación de horarios.
Teniendo los grupos hechos para todas las asignaturas, las prácticas se comienzan de inmediato sin los retrasos que se ocasionaban anteriormente.

El trabajo de módulo permite coordinar diferentes asignaturas y hacer trabajos comunes de modo que los objetivos son compartidos y las competencias reforzadas. Esto implica la realización de un trabajo en un grupo "grande" con sus ventajas y desventajas.

Otro de los requisitos exigidos es la realización de una presentación formal y la defensa de un proyecto.

En general, todos los alumnos han presentado el trabajo de módulo, la gran mayoría ha superado este trabajo con bastante éxito.

Se llevó a cabo una reunión intermedia por parte de los profesores con cada grupo de alumnos a la hora de elegir el concepto definitivo, así los alumnos no perderían tanto tiempo en este punto, actualmente tienen que concertar una reunión con cada profesor, y además los objetivos y alcance de cada una de las asignaturas quedarian claros entre profesores y alumnos.

Se adelantó el inicio del trabajo de módulo para evitar que se retrase todo el trabajo y no se solape con otros trabajos individuales o trabajos de asignatura.

Se permitió la calificación fuera del módulo, por partes separadas de cada asignatura y para alumnos que solo tienen una o dos asignaturas de módulo, además de poder guardar partes de un año a otro como por ejemplo las practicas.

\section{Puntos débiles}

Los alumnos se apuntan a los grupos en función de la preferencia de horarios, pero son ajustados por los profesores en función de las asignaturas en las que están matriculados. Se ha detectado que algunos grupos no son homogéneos y se producen desequilibrios en las cargas de trabajo.

Se ha detectado que es necesario un esfuerzo extra en la coordinación del módulo entre los profesores, sobre todo en la gestión del proyecto común de los alumnos.

ARBOR Vol. 187 Extra 3 diciembre [2011] 231-236 ISSN: 0210-1963 
A pesar de adelantar el inicio del proyecto los alumnos empiezan algo tarde y se juntan trabajos de varias asignaturas al final de modo que el trabajo del módulo les quita excesivas horas para hacer el resto de trabajos de asignatura y estudio personal.

Se debería valorar con unos criterios más definidos la consecución de los objetivos del proyecto de módulo, de las competencias, así como de las presentaciones y el trabajo.

\section{Acciones de mejora}

Facilitar que los grupos los hagan los propios alumnos, de modo que ellos se responsabilicen de la coordinación de su grupo de trabajo en el proyecto.

Buscar algún sistema para penalizar a las "rémoras" que existen en algunos de los grupos.

Insistir en llevar a cabo una reunión de coordinación entre profesores a mitad de cuatrimestre, para tratar de anticipar posibles desviaciones en alcanzar los objetivos, reorientar grupos, etc.

Es necesario crear una rúbrica o sistema de evaluación con índices para poder tener una evaluación objetiva de los proyectos y trabajos.

Se debería tomar algunos criterios comunes para las personas que no hagan el trabajo de módulo porque solo tienen una asignatura y si se guarda la nota de un año para otro.

\section{Condicionantes para la mejora}

Que se puedan adelantar algunos trabajos de asignaturas para que el trabajo de modulo se inicie antes, será necesario que los alumnos puedan tener ciertos conocimientos de inmediato para iniciar algunas de las tareas del trabajo de módulo. Será necesario que alguna de las asignaturas reorganice contenidos para que sea posible el inicio inmediato del trabajo de módulo.

\section{Evaluación del módulo por parte de los alumnos}

Al finalizar el cuatrimestre y una vez realizada la evaluación final, se realizó una encuesta entre los alumnos en la que se les pedía su opinión sobre diversos temas relacionados con el módulo, que se exponen a continuación:

Pregunta 1: El primer día de curso se realizó una presentación conjunta del módulo y se realizaron ya los grupos de trabajo. La opinión de los alumnos sobre la organización y gestión de esos grupos no ha sido demasiado buena, ya que piensan que eran demasiado heterogéneos. La opinión de los profesores es similar a la de los alumnos. Para el próximo curso se propone que los grupos de trabajo se creen más tarde, cuando realmente comiencen a desarrollar el trabajo de módulo, para que ya cada alumno sepa con certeza qué asignaturas va a cursar. Los grupos los propondrán ellos, pero con determinados condicionantes para que no haya demasiadas diferencias entre los grupos, y los confirmarán los profesores.

Pregunta 2: Ante la pregunta de cuál ha sido la relación entre los componentes del grupo, la respuesta ha sido mayoritariamente que buena.

Pregunta 3: Posteriormente se les preguntó sobre la coordinación entre los profesores de las distintas asignaturas del módulo. En este caso hay disparidad en las respuestas, desde que buena hasta que mala. El profesorado opina que es un punto en el que debe mejorar y para ello propone varias medidas. En primer lugar se va a modificar el cronograma de las asignaturas, especialmente la de Taller de Diseño II, para que los alumnos puedan comenzar antes el trabajo de módulo. También, y en respuesta a algunos alumnos, se van a realizar dos sesiones de tutorías en los que estén presentes el grupo de alumnos y al menos un profesor de cada una de las cuatro asignaturas.

Pregunta 4: Uno de los objetivos del módulo era dar sentido y contexto a cada una de las asignaturas. Se les preguntó en la encuesta sobre la utilidad que tenía el trabajo del módulo para agrupar todas las asignaturas. Todos los alumnos opinan que es una buena idea, aunque algunos matizan que la carga de trabajo es alta y que tal vez debería eliminarse alguna otra actividad de las asignaturas individuales.

Pregunta 5. Se les preguntó a los alumnos sobre la adecuación de este tema a la temática de las cuatro 
asignaturas. La respuesta en este caso también ha sido dispar. Aproximadamente la mitad de los alumnos opinaba que estaba bien, y la otra mitad sugería otro tipo de problemas, como mobiliario. La opinión de los profesores es que el tema ha sido correcto. La trona es un concepto suficientemente amplio y versátil para la asignatura de Taller de Diseño II, y el hacerla plegable permite desarrollar distintos mecanismos que han sido tratados especialmente en Mecánica y Diseño Asistido por Ordenador. Los planos, tanto generales como de despieces, han sido el tema de trabajo de la asignatura Expresión Gráfica II. Los profesores han detectado que algunos de los mecanismos presentados no estaban desarrollados suficientemente.

Pregunta 6: Se les preguntó sobre si los conocimientos adquiridos en cada asignatura habian sido suficientes para poder realizar el trabajo de módulo. La respuesta general ha sido que sí.
Pregunta 7: Se les pidió su opinión sobre la calidad de la atención personalizada de los profesores a los grupos. En este caso su opinión de que la atención ha sido buena.

Pregunta 8: Se les preguntó sobre la relación de horas de trabajo y el porcentaje de nota en la evaluación. La opinión de los alumnos es que han necesitado muchas más horas de las que luego han contado en la evaluación.

Pregunta 9: Se les pidió su opinión sobre el sistema de evaluación del trabajo de módulo. La respuesta del alumnado es que la evaluación y los porcentajes han sido correctos.

Pregunta 10: Los trabajos se evaluaron dentro de las semanas de exámenes, concretamente en la tercera semana e intercalados entre dos exámenes. Se les preguntó a los alumnos su opinión sobre cuándo deberian evaluarse los trabajos. La respuesta de los alumnos y profesores fue unánime: fuera de la época de exámenes.

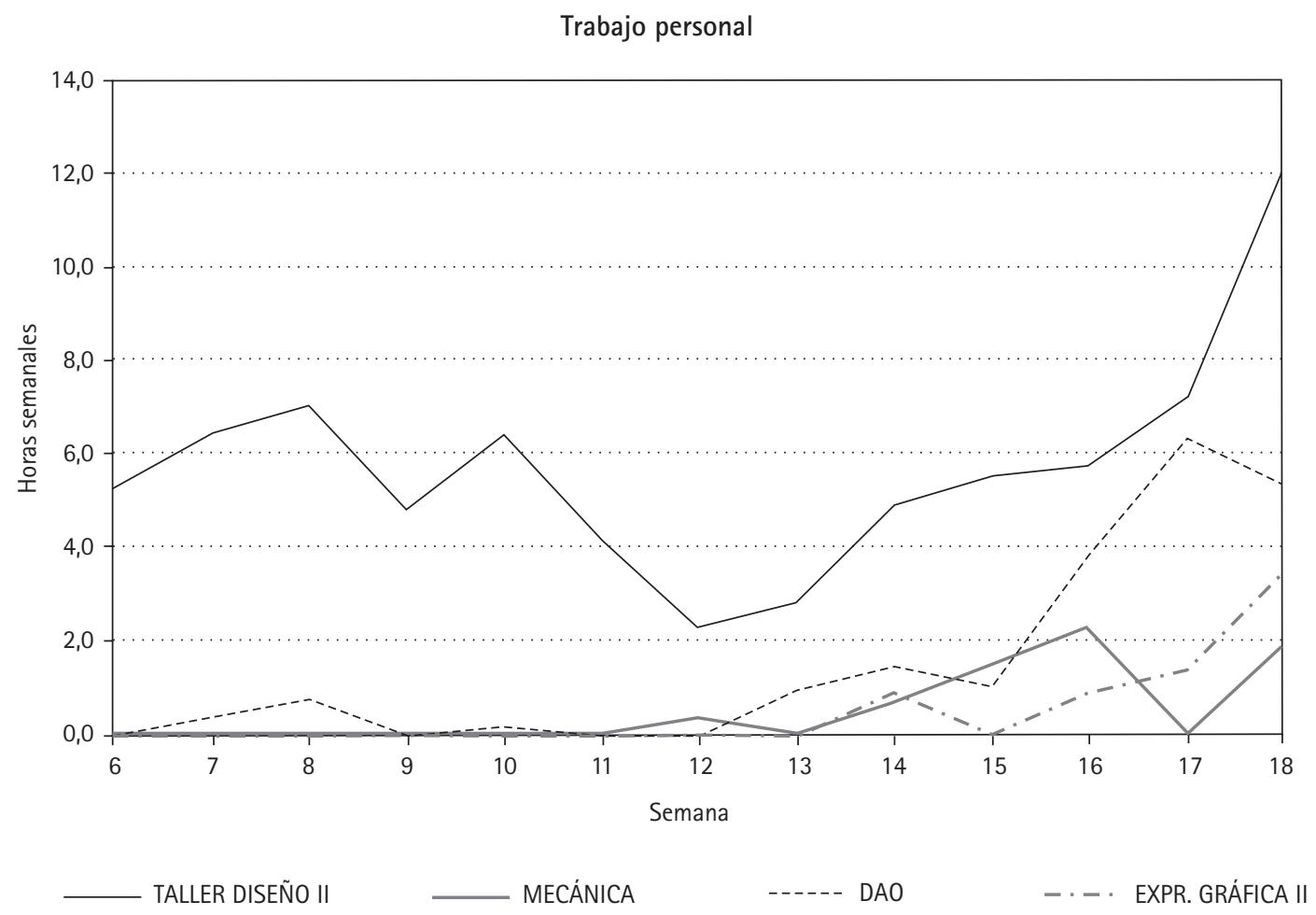

Figura 1. Horas semanales de trabajo personal a lo largo del cuatrimestre. 


\section{Evaluación de la carga de trabajo}

Con el fin de evaluar objetivamente el número de horas que cada alumno ha dedicado al trabajo de módulo, se propuso a los alumnos la realización de una hoja de cálculo en la que fueran rellenando, cada semana, las horas individuales y de trabajo de grupo dedicadas al módulo, indicando asimismo a qué asignatura respondía esa parte del trabajo. En la gráfica 1 se muestra, a modo de resumen, el número de horas promedio empleado y se muestra también el número de horas de trabajo individual y el número de horas empleado en trabajar en grupo.

Se observa que la mayor carga de trabajo es la dedicada a la asignatura Taller de diseño II, con un total de 171.3 horas. En esta asignatura el trabajo de módulo es muy importante, y tiene un alto valor en el porcentaje de evaluación final, por lo que esta carga de trabajo no es excesiva. El número de horas totales empleados para el resto de las asignaturas es de 51.2. Aunque, según los alumnos, la mayor parte de horas las han dedicado a Diseño Asistido por Ordenador, hay que comentar que la mayor parte del trabajo realizado en esta asignatura es básico y necesario para poder realizar las partes asociadas a Mecánica y a Expresión Gráfica II, por lo que una proporción no despreciable de las horas dedicadas a Diseño Asistido por Ordenador pueden considerarse compartidas con las otras dos asignaturas.

\section{Conclusiones globales}

La conclusión general es que los alumnos alcanzan un nivel superior al obtenido con la anterior titulación de ingeniería técnica y adquieren una serie de capacidades como el trabajo en grupo y la capacidad de realizar defensas orales de un proyecto gracias al proyecto de módulo, aunque es necesaria una buena coordinación entre profesores. También se ha constatado una menor carga global de trabajo para los alumnos aunque ésta se concentra más a final de curso.

\section{REFERENCIAS}

"El Camino hacia Bolonia: Experiencias de Innovación Docente", Revista de la UAM (2010).
López, J. A. (2010): La universidad (y el proceso de Bolonia), Madrid, Rustica. 